\title{
Point Prevalence Survey of Antimicrobial Use in Four Tertiary Care Hospitals in Mexico
}

\author{
Federico A Zumaya-Estrada' \\ Alfredo Ponce-de-León- \\ Garduño ${ }^{2}$ \\ Edgar Ortiz-Brizuela $\mathbb{D}^{2}$ \\ Juan Carlos Tinoco-Favila ${ }^{3}$ \\ Patricia Cornejo-Juárez ${ }^{4}$ \\ Diana Vilar-Compte ${ }^{4}$ \\ Alejandro Sassoé-González ${ }^{5}$ \\ Pedro Jesus \\ Saturno-Hernandez $\mathbb{D}^{6}$ \\ Celia M Alpuche-Aranda' \\ 'Center for Infectious Diseases Research \\ (CISEI), National Institute of Public \\ Health (INSP), Cuernavaca, C.P. 62100, \\ Morelos, México; ${ }^{2}$ Infectology \\ Department, National Institute of \\ Medical Sciences and Nutrition "Salvador \\ Zubirán”, Ciudad de México, C.P. I4080, \\ México; ${ }^{3}$ Infectology Department, \\ Hospital General 450, Secretary of \\ Health of Durango, Durango, C.P. 34206, \\ Durango, México; ${ }^{4}$ Infectious Diseases \\ Department, National Institute of Cancer \\ (INCan), Ciudad de México, C.P. I4080, \\ México; ${ }^{5}$ Epidemiological Intelligence \\ Unit, High Specialty Regional Hospital of \\ Ixtapaluca, Estado de México, C.P. 56530, \\ México; ${ }^{6}$ Center for Evaluation and \\ Surveys Research (CIEE), National \\ Institute of Public Health (INSP), \\ Cuernavaca, C.P. 62100, Morelos, México
}

Correspondence: Celia M Alpuche-Aranda Center for Infectious Diseases Research (CISEI), National Institute of Public Health (INSP), Cuernavaca, C.P. 62100, Morelos,

México

Tel +52 777-329-3000 ext. 2I 01

Email celia.alpuche@insp.mx

Pedro Jesus Saturno-Hernandez

Center for Evaluation and Surveys Research

(CIEE), National Institute of Public Health

(INSP), Cuernavaca, C.P. 62100, Morelos,

México

Tel +52 777-329-3000 ext. 4332

Email pedro.saturno@insp.mx
Purpose: To describe the antimicrobial use in four tertiary care hospitals in Mexico.

Patients and Methods: Point prevalence surveys (PPSs) were conducted on medical records of hospitalized patients with prescribed antimicrobials (AMs) in four tertiary care hospitals in Mexico in 2019. Prevalence estimates and descriptive statistics were used to present the collected data on antimicrobial prescribing and microbiological studies.

Results: The prevalence of patients with prescribed AMs among the hospitals ranged from $47.1 \%$ to $91.3 \%$. Antibiotics for systemic use (J01s) were the most prescribed $(84.6 \%$, [95\% CI: 81.5-87.3]), mainly extended-spectrum J01s: third-generation cephalosporins $19.8 \%$ [95\% CI: 16.8-23.1], and carbapenems 17.0\% [95\% CI: 14.2-20.2]. Antibiotic treatments were largely empirical, with no planned duration or review dates. The ceftriaxone use was excessive and prolonged. No formal reference guidelines for antimicrobial prescribing were available in the hospitals. Multidrug-resistant Escherichia coli and ESKAPE pathogens were identified in all hospitals.

Conclusion: This study describes the extensive use of antimicrobials and broad-spectrum antibiotics for systemic use in Mexican hospitals, along with the presence of resistant pathogens to the antibiotics frequently used in the hospitals surveyed.

Keywords: PPSs, antimicrobial use, antibiotics, Mexican hospitals

\section{Introduction}

Antimicrobials (AMs) are the pharmacological health-care standard against infectious diseases. Any usage of antimicrobials, mainly antibacterials (antibiotics) contributes to the selection of antimicrobial resistance (AMR), but their misuse and overuse worsens it. ${ }^{1}$ Antimicrobial-resistant infections cause serious illnesses and prolonged hospital stays, increasing health-care costs, treatment failures, and morbi-mortality rates. ${ }^{2,3}$

In the US alone, a conservative estimate indicates that more than two million people every year are affected with antimicrobial-resistant infections, with at least 23,000 dying as a result. ${ }^{4}$ Although it has been estimated that a continued rise in AMR by 2050 would lead to 10 million deaths every year worldwide, ${ }^{5}$ the fact that infectious diseases can no longer be treated with antimicrobials could represent an even bleaker future. Given the serious threat that AMR represents to global public health, the efforts to reduce the irrational antibiotic use are becoming an increasing priority. ${ }^{6}$

Inadequate antibiotic therapies are common in hospitals regarding the agent's selection for microorganism type, routes of administration or treatments duration. ${ }^{7}$ Thus, the majority of hospitalized patients are exposed either to potentially serious 
adverse effects or the acquisition of resistant or difficult-to -treat pathogens (ie, Clostridium difficile), without any therapeutic benefit. ${ }^{8,9}$

Selection of antibiotic-resistant bacteria in hospitals may be aggravated by the frequent extended-spectrum antibiotics usage (eg, cephalosporins and carbapenems), and concentrated in common causal agents of hospitalassociated infections (HAIs), such as those of the ESKAPE group (Enterococcus faecium, Staphylococcus aureus, Klebsiella pneumoniae, Acinetobacter baumannii, Pseudomonas aeruginosa, and Enterobacter spp), driven to a vicious cycle of greater use of extended-spectrum antibiotics. ${ }^{10,11}$

The World Health Organization (WHO) global action plan on AMR seeks to strengthen surveillance and optimize antimicrobial prescribing. ${ }^{6}$ These actions are promoted in hospitals with antimicrobial stewardship programs (ASPs) through interventions to improve clinical outcomes, ensure the antimicrobial therapy's costeffectiveness, and reduce unintended use consequences. ${ }^{12}$ However, the needed effort, coordination, and resources for active monitoring of antimicrobial prescribing behavior in hospitals are high.

In Mexico, there is still little information on the antimicrobial use in general, and in the hospital environment in particular. Earlier studies focused on national consumption of antibiotics, based on retail sales. ${ }^{13}$ More recently, the main focus has been on the epidemiology of HAIs, which usually report also some valuable data on antimicrobial use. ${ }^{14,15}$ One of these studies, using the one-day point prevalence approach for HAIs, included four Mexican hospitals within a study in four Latin-American countries. This study reported that Mexican hospitals had the highest prevalence of patients on antibiotics (59.3\%) among the four countries, and a relatively extensive use of third-generation cephalosporins, and carbapenems. ${ }^{14}$ A related topic for research has been the bacterial pathogens and AMR. ${ }^{16,17}$ These studies report a high frequency of AMR in both Gram-negative and Gram-positive bacteria, including high levels of multi-drug resistance in some strains. Besides, the antibiotic use for surgical prophylaxis is of particular concern. Several studies have shown the worrisome low frequency of good clinical practices in this regard. In one particular hospital, the level of compliance with international guidelines for surgical prophylaxis was $6.5 \%{ }^{18}$ In another study, also in a single hospital, only $3.9 \%$ of surgical patients received the recommended antimicrobial prophylaxis. ${ }^{19}$ Even after a multi-centric improvement initiative, inappropriate prophylaxis regimens were found in $75.3 \%$ of surgical patients, and inappropriate dosages in $51.2 \%{ }^{20}$ To date, in spite of this worrisome panorama, there are not official programs to analyze and improve the patterns of antibiotic use in Mexico. Besides, comprehensive studies on the characteristics of antimicrobial prescription and use are also lacking.

Point prevalence surveys (PPSs) are a useful method to study the antimicrobial prescription patterns in order to identify objectives for optimization on antibiotic use. ${ }^{21-23}$ Therefore, the Global-PPS initiative and WHO (WPPS) have proposed standardized methodologies to conduct PPSs in hospitals. ${ }^{23,24}$

The aim of this study is to describe the antimicrobial use in four tertiary care hospitals in Mexico by means of PPSs.

\section{Materials and Methods} PPS Protocol and Hospitals Characteristics

The protocol for this study was based on the Global-PPS and the WPPS methodologies ${ }^{23,24}$ with a few modifications previously standardized. ${ }^{25}$ Four third-care hospitals were selected for the PPSs. Hospitals will be described with anonymous nomenclature as $\mathrm{H} 1, \mathrm{H} 2, \mathrm{H} 3$ and $\mathrm{H} 4$.

$\mathrm{H} 1$ and $\mathrm{H} 2$ are highly specialized national referral institutions located in Mexico City, with 167 and 119 beds, respectively. Annually, each one provides healthcare to around five thousand patients. $\mathrm{H} 3$ is a referral hospital located in the State of Mexico, with 246 beds, and each year provides specialized clinical and surgical care to nearly six thousand patients. H4 is a general hospital located in a state in the northeast of Mexico, it has 120 beds and provides specialized medical care to approximately 4500 low-income patients. Regarding antimicrobial stewardship activities in the hospitals surveyed, H1 reported educational activities on antibiotics and pharmacovigilance interventions; $\mathrm{H} 2$ has implemented some interventions to improve the antibiotic prescribing; $\mathrm{H} 3$ reported having surveillance activities of resistant-ESKAPE pathogens. However, no hospital reported to have specific funding or personnel for these activities, neither documents or records to support any formally established ASP were found or available. No data were available on antimicrobial stewardship activities or AMR surveillance in H4.

\section{Observer Team}

The PPSs were conducted in all hospitals by the same multidisciplinary observer team, integrated by three 
professionals with expertise in health surveys, supported by the principal investigators and the health-care providers of the hospitals. The observers were trained for one week on the PPS method, the study's aims, the characteristics and content of the questionnaires, and the procedures for data collection and management. The team was under the direct supervision of at least one principal investigator during the pilot studies and the PPSs.

\section{PPS Pilot Studies}

Pilot studies were conducted in each hospital at least one week in advance of the PPSs to identify the data sources and to allow corrective action. Anonymized data were collected in REDCap from two questionnaires based on the variables set proposed by the WPPS. Each pilot study included 15 patients hospitalized at 8:00 am on a particular day. Inter-observer agreement was assessed on the total variables of both questionnaires.

The preparation, coordination, and data collection difficulties faced during our pilot studies, as well as the identified gaps in WPPS guidance to address common challenges for PPS implementation are reported in our previous work. ${ }^{25}$

\section{PPSs in the Hospitals}

The PPSs were conducted in August, October, November, and December 2019. Data were collected from patients with at least one AM prescribed, any age, hospitalized at 8:00 am on the day of the surveys in the medical and surgical wards, and the intensive-care units (ICUs). Data from outpatients or patients with surgery on the same day of the PPSs were not included.

Data collection was from 8:00 am to 8:00 pm, any day between Monday and Friday for patient selection, but data corroboration for selected patients was followed for two weeks. Written data on antimicrobial prescribing found in prescription sheets, medical notes, Kardex and nursing notes, nutrition records, hospital census, surgical scheduling sheets, and laboratory reports were collected.

Anonymized recorded data on the patients' age, sex, admission date, clinical diagnoses, wards/units of care, invasive devices presence, and the type of indication (therapeutic or prophylactic) were collected. The patients' type of infection (HAIs or community-acquired infections (CAIs)) defined in the medical records was collected. The observer team corroborated the onset dates of infections' first signs or symptoms after admission in the patients' medical records. When these dates were not found, the date the antimicrobial treatment was started or the date the first sample was taken was considered according to the WPPS recommendations for classification of HAI versus CAI. The recorded data in the medical records on microbiological studies and antimicrobial susceptibility testing (AST) also was collected.

The name, number, and ATC codes (Anatomical Therapeutic Chemical classification system) of the AMs prescribed were collected, ${ }^{26}$ as well as the reasons for the antimicrobial prescription, the type of treatments (empirical or targeted), routes of administration, doses, duration and review dates of treatments. Data on topical or ophthalmologic AMs were not included.

The observer team was instructed to notify the principal investigators when the medical record data were not clear to them or when there were doubts about capturing the information.

\section{Data Management and Analysis}

The PPSs data collected were exported to the SPSS v25 statistical package for cleaning and analysis. ${ }^{27}$ The prevalence by hospital, wards and units was defined as the percentages of the number of patients with AMs, and antibiotics for systemic use (ATC level 2: J01s) prescribed, divided by the total number of patients admitted on the day of the PPSs. As appropriate, descriptive statistics (frequencies, proportions, and ranges between hospitals surveyed) were used to examine the data on patients, clinical diagnoses, prescribed agents (ATC levels 3, 4 and 5), routes of administration, types of indication, infection and treatments, surgical prophylaxis dose-day schedules, microbiological studies, and ASTs, by hospital, wards and units. The $95 \%$ confidence intervals $[95 \% \mathrm{CI}]$ were calculated using GraphPad QuickCalcs. ${ }^{28}$

\section{Results}

\section{Most of the Hospitalized Patients Received AMs}

PPSs detected a total of 525 patients, and 348 (66.3\%, [95\% CI: 62.1-70.2]) of them were receiving AMs in the four hospitals. The mean age of the patients with AMs was between 37 and 50 years. All patients were adults in $\mathrm{H} 1$. The highest children proportion was $18.6 \%$ in H3, including a neonatal ward (4.1\%). The proportions by gender were similar, except for $\mathrm{H} 4$ where more male patients were found (68.2\%) (Annex 1). Pneumonia and intraabdominal sepsis were the most frequent clinical 
diagnoses of patients receiving AMs. Nonetheless, the clinical diagnosis was documented in less than half of the patients in two of the four hospitals (Annex 2).

The prevalence of patients with AMs prescribed in the hospitals surveyed ranged from $47.1 \%$ to $91.3 \%$. The prevalence of patients who had received J01s was in similar ranges, from $46.2 \%$ to $82.6 \%$. The prevalence of patients with AMs prescribed among the wards/units ranged from $55.6 \%$ to $89.4 \%$, almost equal to the range of patients who had received J01s (55.1\% to $89.4 \%$ ).

The volume of prescription was high in three of the hospitals (H1-H3), the medical wards, and ICUs, with prescription of three or more AMs by patient (Table 1).

\section{Extensive Use of AMs Was Based on Empirical Decisions}

The average number of days of AM treatments in patients ranged from 4.1 to 7.7 days among hospitals, and 5.9 (medical wards) to 7.5 days (ICUs) among wards/units. At the day of PPSs data collection, between $61.9 \%$ and 90.5\% of patients with AMs were treated empirically, and between $60.5 \%$ and $91.8 \%$ of those who had received J01s. Besides, between $81.1 \%$ and $91.9 \%$ of the AMs were administered parenterally. Parenteral J01s was above $90 \%$ in all hospitals. In addition, almost all AMs and J01s used in the ICUs and surgical wards were administered parenterally (Table 2).

The AMs and J01s indication use were documented in most cases. However, none of the hospitals was documented a planned duration and review dates of the antibiotic therapies. There was also no evidence of formal reference guidelines for antimicrobial prescribing in the four hospitals, but in three of them there were specialists qualified in infectious diseases with different authority levels to control or recommend the prescription of some antibiotics.

\section{The J0Is Was the Main Group of AMs Used in Hospitalized Patients}

Overall, six hundred and six prescriptions of AMs were issued, and $84.6 \%$ [95\% CI: $81.5-87.3$ ] of these corresponded to J01s $(\mathrm{n}=513)$, mainly beta-lactam antibiotics (ATC level 3: J01D). Almost all AMs used in H4, the surgical wards, and ICUs were J01s. Other AM groups (ATC level 2) prescribed in the hospitals in much smaller proportions were: antimycotics (J02: 7.9\%, [95\% CI: 6.0 10.3]), and antivirals for systemic use (J05: 5.8\%, [95\% CI: 4.1-7.9]), anti-diarrheal, intestinal anti-inflammatory /anti-infective agents (A07: $0.8 \%,[95 \%$ CI: 0.2-1.9]), anti-mycobacterials (J04: $0.5 \%$, [95\% CI: $0.1-1.5]$ ), and anti-parasite agents (P02C: $0.3 \%,[95 \%$ CI: $0.1-1.2]$ ) (Table 3).

The J01s were mainly used for therapeutic indications (ie, HAIs, and CAIs) in three of the hospitals (Table 2). The use of J01s for medical prophylaxis ranged from $4.2 \%$ to $27.3 \%$, whereas, for surgical prophylaxis it ranged from $14.4 \%$ to $25.0 \%$. All or most of the surgical prophylaxis in the hospitals comprised

Table I Prevalence and Volume of Use of AMs and J0Is in the Hospitals and Wards/Units Surveyed

\begin{tabular}{|c|c|c|c|c|c|c|c|c|}
\hline & & \multicolumn{4}{|c|}{ Hospitals } & \multicolumn{3}{|c|}{ Wards/Units - All Hospitals } \\
\hline & & HI & $\mathrm{H} 2$ & H3 & H4 & Medical & Surgery & ICUs \\
\hline & & $\begin{array}{c}n(\%) \\
{[95 \% \mathrm{Cl}]}\end{array}$ & $\begin{array}{c}n(\%) \\
{[95 \% \mathrm{Cl}]}\end{array}$ & $\begin{array}{c}n(\%) \\
{[95 \% \mathrm{Cl}]}\end{array}$ & $\begin{array}{c}n(\%) \\
{[95 \% \mathrm{Cl}]}\end{array}$ & $\begin{array}{c}n(\%) \\
{[95 \% \mathrm{Cl}]}\end{array}$ & $\begin{array}{c}n(\%) \\
{[95 \% \mathrm{Cl}]}\end{array}$ & $\begin{array}{c}n(\%) \\
{[95 \% \mathrm{Cl}]}\end{array}$ \\
\hline Prevalence rates & $\begin{array}{l}\text { Patients with } \\
\text { AMs } \\
\text { Patients with } \\
\text { Jols }\end{array}$ & $\begin{array}{c}84(91.3) \\
{[83.5-95.7]} \\
76(82.6) \\
{[73.5-89.1]}\end{array}$ & $\begin{array}{c}56(47.1) \\
{[38.3-55.9]} \\
55(46.2) \\
{[37.5-55.1]}\end{array}$ & $\begin{array}{c}145(64.7) \\
{[58.2-70.7]} \\
143(63.8) \\
{[57.3-69.8]}\end{array}$ & $\begin{array}{c}63(70.0) \\
{[59.8-78.5]} \\
61(67.7) \\
{[57.5-76.5]}\end{array}$ & $\begin{array}{c}174(69.0) \\
{[63.0-74.4]} \\
162(64.3) \\
{[58.1-69.9]}\end{array}$ & $\begin{array}{c}115(55.6) \\
{[48.7-62.1]} \\
114(55.1) \\
{[48.2-61.7]}\end{array}$ & $\begin{array}{c}59(89.4) \\
{[79.4-95.0]} \\
59(89.4) \\
{[79.4-95.0]}\end{array}$ \\
\hline Patients per number of AMs & $\begin{array}{l}\text { One AM } \\
\text { Two AMs } \\
\geq \text { Three AMs }\end{array}$ & $\begin{array}{l}16(19.0) \\
24(28.6) \\
44(52.4)\end{array}$ & $\begin{array}{l}12(21.4) \\
16(28.6) \\
28(50.0)\end{array}$ & $\begin{array}{l}38(26.2) \\
41(28.3) \\
66(45.5)\end{array}$ & $\begin{array}{l}27(42.9) \\
25(39.7) \\
\mathrm{II}(17.5)\end{array}$ & $\begin{array}{l}39(22.4) \\
50(28.7) \\
85(48.9)\end{array}$ & $\begin{array}{l}43(37.4) \\
38(33.0) \\
34(29.6)\end{array}$ & $\begin{array}{l}\text { II (18.6) } \\
18(30.5) \\
30(50.8)\end{array}$ \\
\hline $\begin{array}{l}\text { Average number of } \\
\text { prescriptions per patient }\end{array}$ & $\begin{array}{l}\text { AMs } \\
\text { JOls }\end{array}$ & $\begin{array}{l}1.9[164 / 84] \\
1.4[120 / 84]\end{array}$ & $\begin{array}{l}2.0[112 / 56] \\
1.5[86 / 56]\end{array}$ & $\begin{array}{l}1.6[234 / 145] \\
1.5[217 / / 45]\end{array}$ & $\begin{array}{l}1.5[96 / 63] \\
1.4[90 / 63]\end{array}$ & $\begin{array}{l}1.8[3 / 5 / 174] \\
1.4[239 / 174]\end{array}$ & $\begin{array}{l}1.5[176 / 115] \\
1.5[171 / 115]\end{array}$ & $\begin{array}{l}1.9[115 / 59] \\
1.7[103 / 59]\end{array}$ \\
\hline
\end{tabular}

Notes: AM prescribed groups (ATC level 2): Antibacterials for systemic use (J0I); Antimycotics for systemic use (J02); Antimycobacterials (J04); Antivirals for systemic use (05); Anti-diarrheal, intestinal anti-inflammatory/anti-infective agents (A07); Anti-parasite agents (P02C). HI does not have surgery ward. H4 did not have patients admitted in ICU.

Abbreviations: AMs, antimicrobials (all identified groups); J0Is, antibacterials for systemic use. 


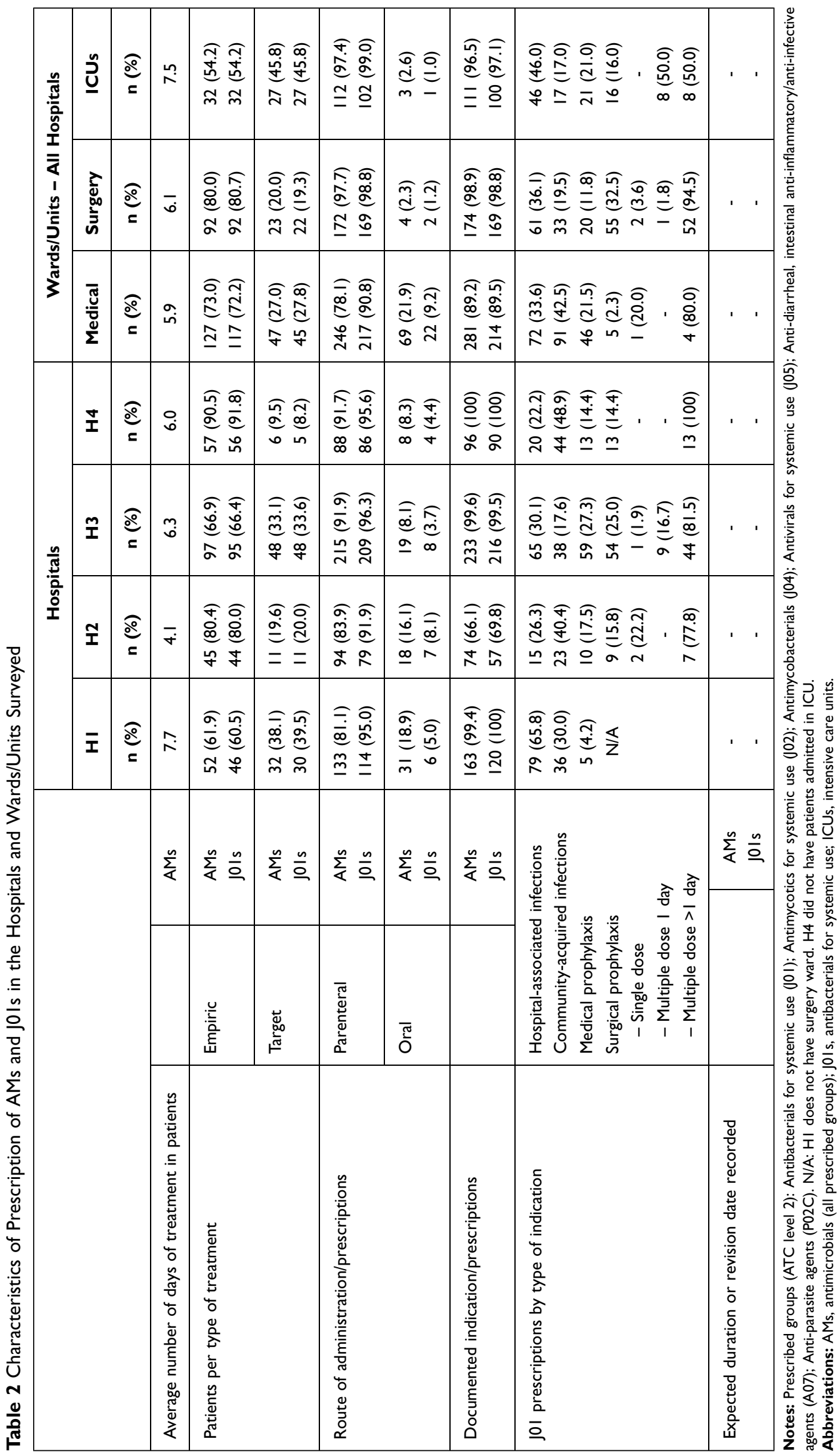




\begin{tabular}{|c|c|c|c|c|c|c|c|c|c|c|c|c|c|c|}
\hline \multicolumn{2}{|l|}{$\begin{array}{l}\overline{0} \\
\text { 음 }\end{array}$} & 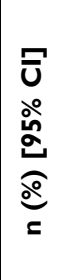 & 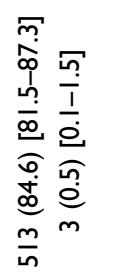 & 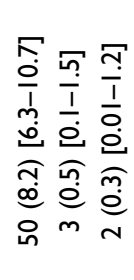 & 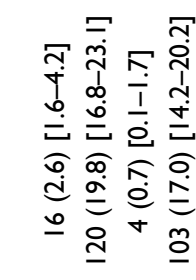 & 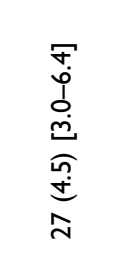 & 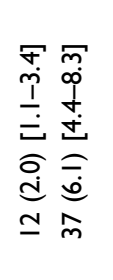 & 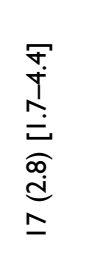 & 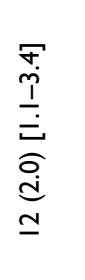 & 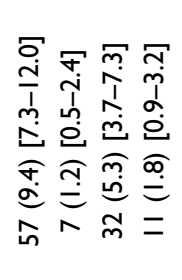 & 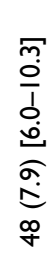 & 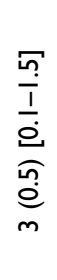 & 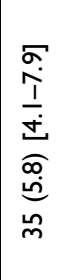 & 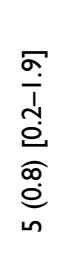 \\
\hline \multirow{3}{*}{ 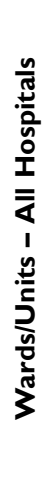 } & $\underline{\tilde{u}}$ & 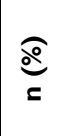 & 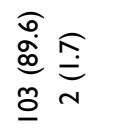 & 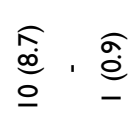 & 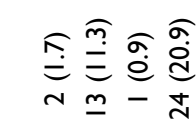 & $\underset{a}{\stackrel{0}{\infty}}$ & $\begin{array}{l}\hat{\sigma} \\
\hat{e} \\
-\hat{0} \\
-1\end{array}$ & $\underset{\overline{0}}{\stackrel{0}{n}}$ & 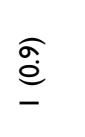 & 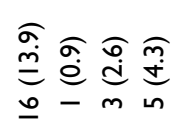 & $\begin{array}{l}\underset{\widehat{c}}{\infty} \\
\infty \\
0\end{array}$ & . & 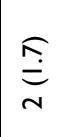 & \\
\hline & 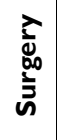 & $\underset{s}{\stackrel{\bigodot}{9}}$ & 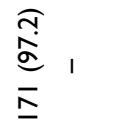 & 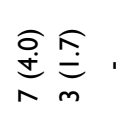 & 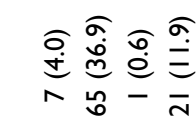 & , & 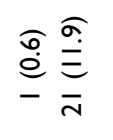 & $\underset{r}{\stackrel{o}{+}}$ & $\underset{r}{\stackrel{\sigma}{\dot{+}}}$ & 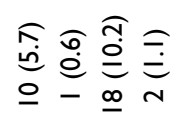 & $\underset{m}{\stackrel{F}{E}}$ & . & ' & \\
\hline & $\begin{array}{l}\overline{\frac{\pi}{\pi}} \\
\frac{\Phi}{\Sigma}\end{array}$ & $\stackrel{\bigodot}{\bigodot}$ & 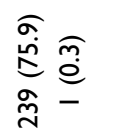 & 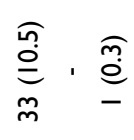 & 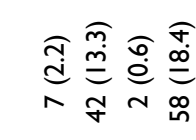 & $\begin{array}{l}\hat{\kappa} \\
\stackrel{\infty}{0} \\
\underline{\infty}\end{array}$ & 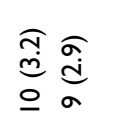 & $\underset{m}{\stackrel{o}{\dot{\rho}}}$ & 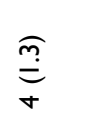 & 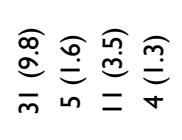 & $\widehat{\overline{\bar{\Xi}}}$ & $\underset{m}{\stackrel{o}{\leftrightarrows}}$ & $\stackrel{\substack{\tilde{n} \\
\stackrel{m}{\varrho}}}{\stackrel{m}{m}}$ & 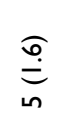 \\
\hline \multirow{4}{*}{ 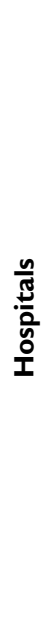 } & $\stackrel{\mathbb{I}}{I}$ & $\underset{=}{\stackrel{\overbrace{}}{\bigodot}}$ & 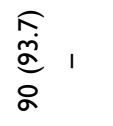 & , $\stackrel{\overline{\bar{m}}}{\bar{m}}$. & 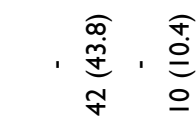 & . & 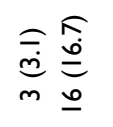 & $\widehat{\overline{\bar{m}}}$ & $\underset{\sim}{\stackrel{\bar{d}}{\sim}}$ & $\underset{\infty}{\widehat{\infty}} \underset{\infty}{\infty}, \underset{\bar{m}}{\widehat{m}}$, & $\underset{\sim}{\stackrel{i}{d}}$ & 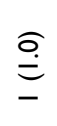 & 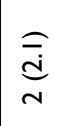 & ' \\
\hline & $\stackrel{m}{I}$ & $\stackrel{\bigodot}{\bigodot}$ & 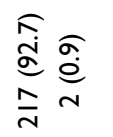 & $\frac{\widehat{\sigma}}{\dot{e}}$, & 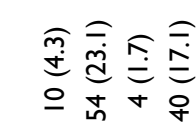 & $\underset{0}{\stackrel{0}{d}}$ & 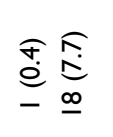 & $\stackrel{\widehat{\overline{\underline{c}}}}{\underline{\simeq}}$ & $\underset{0}{\stackrel{0}{d}}$ & 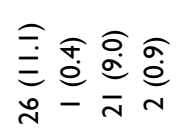 & $\begin{array}{l}a \\
\stackrel{\infty}{0} \\
a\end{array}$ & . & 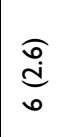 & $\underset{-}{\stackrel{f}{d}}$ \\
\hline & $\tilde{\mathbf{I}}$ & $\underset{=}{\stackrel{\overbrace{}}{9}}$ & $\begin{array}{l}\widehat{\infty} \\
\stackrel{0}{0} \\
\stackrel{0}{0} \\
\infty \\
\infty\end{array}$ & $\begin{array}{l}\stackrel{\sigma}{\stackrel{\sigma}{\sigma}}, \\
=\end{array}$ & 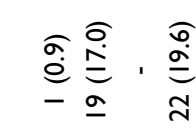 & $\underset{\infty}{\overline{0}}$ & $\underset{\sim}{\stackrel{\infty}{\rightleftarrows} \underset{m}{\stackrel{i}{d}}}$ & $\underset{-}{\stackrel{a}{e}}$ & $\underset{\sim}{\stackrel{\infty}{\infty}}$ & 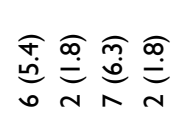 & 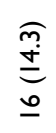 & $\begin{array}{l}\text { å } \\
\text { e }\end{array}$ & 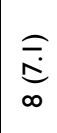 & $\underset{-}{\stackrel{\sigma}{e}}$ \\
\hline & $\overline{\mathbf{I}}$ & $\stackrel{\bigodot}{\bigodot}$ & 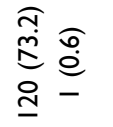 & 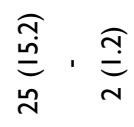 & 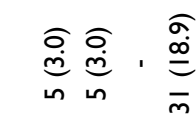 & 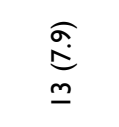 & 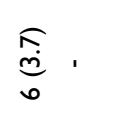 & 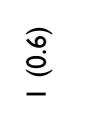 & $\underset{\sim}{\stackrel{\overbrace a}{\leftrightarrows}}$ & 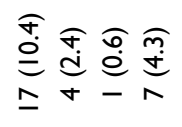 & 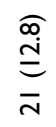 & 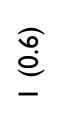 & $\begin{array}{l}\stackrel{\sigma}{\underline{\sigma}} \\
\bar{\sigma} \\
\underline{\sigma}\end{array}$ & $\underbrace{\infty \frac{\infty}{=}}_{m}$ \\
\hline & & 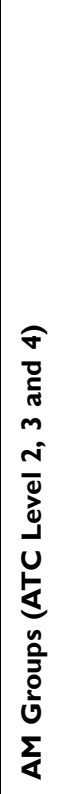 & 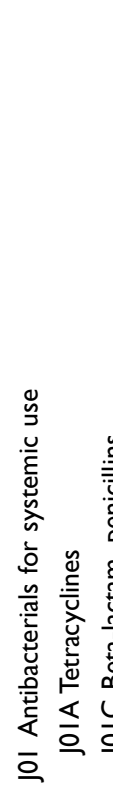 & 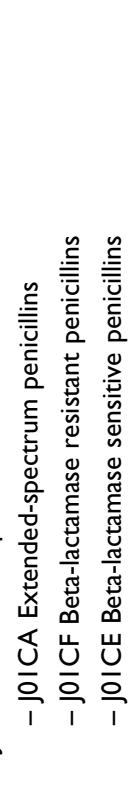 & 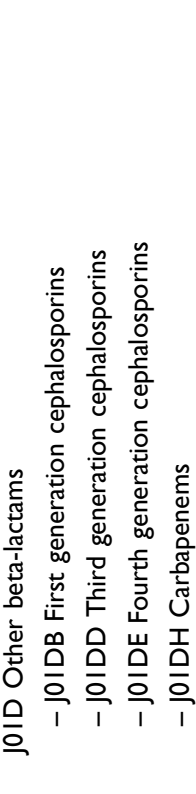 & 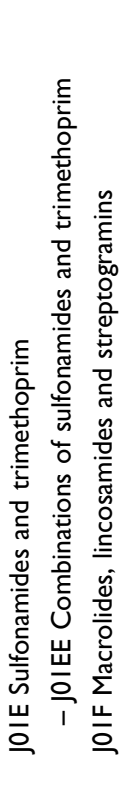 & 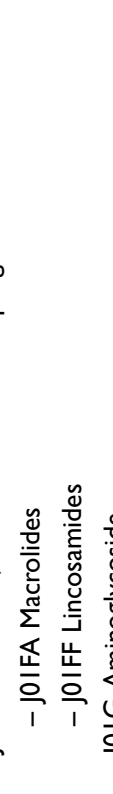 & 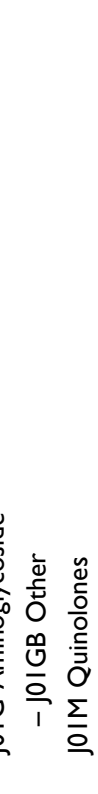 & 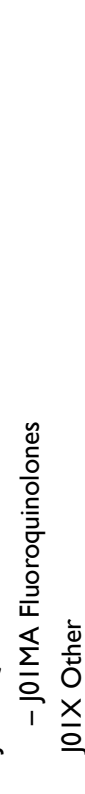 & 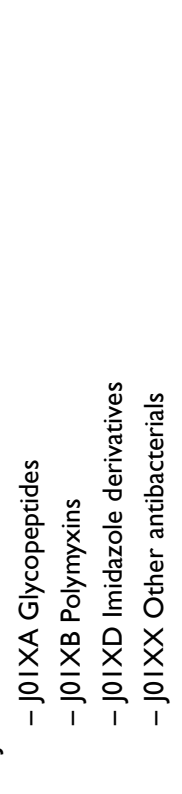 & 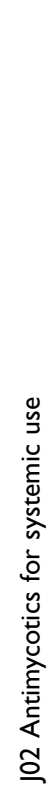 & 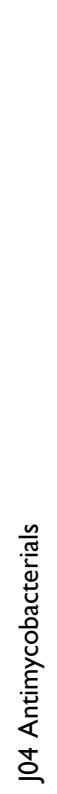 & 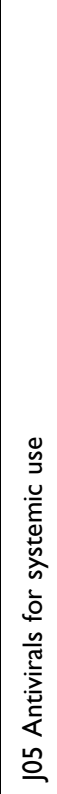 & 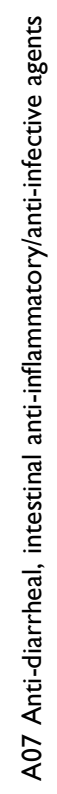 \\
\hline
\end{tabular}




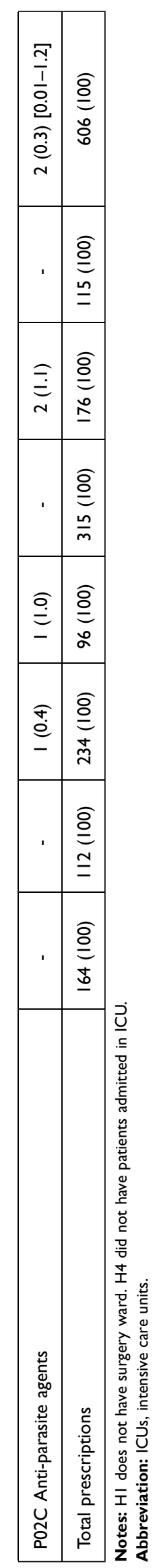

multiple applications of antibiotics for more than one day. Ninety-four percent of surgical prophylaxis was under this schedule (Table 2).

\section{Numerous Prescriptions of Four Groups of Extended-Spectrum J0Is}

Regarding the total prescriptions of AMs $(n=606)$, the highest proportion corresponded to the J01 groups (ATC level 4): third-generation cephalosporins $19.8 \%$ [95\% CI: 16.8-23.1], carbapenems $17.0 \%$ [95\% CI: 14.2-20.2], glycopeptides $9.4 \%$ [95\% CI: 7.3-12.0], and extendedspectrum penicillins $8.2 \%$ [95\% CI: 6.3-10.7] (Table 3). These four groups of antibiotics represented more than $60 \%$ of total J01 prescriptions in the hospitals, and wards/units surveyed (Figure 1). The third-generation cephalosporins were the most prescribed AM group in $\mathrm{H} 3, \mathrm{H} 4$, and within the surgical wards, while the carbapenems were most used in H1, H2, the medical wards, and ICUs (Table 3). The five most used J01s (ATC level 5) in the hospitals were ceftriaxone $21.0 \%$ [95\% CI: $17.7-24.8$ ], meropenem $14.2 \%$ [95\% CI: 11.4-17.5], vancomycin $11.1 \%$ [95\% CI: 8.6-14.1], piperacillin $8.0 \%$ [95\% CI: 5.9-10.6], and clindamycin $7.2 \% \quad[95 \%$ CI: $5.2-9.8]$. Ceftriaxone prescriptions accounted for almost half of total J01 prescriptions in H4. Ceftriaxone was also the most used antibiotic in the surgery and the medical wards. A similarly high proportion of meropenem prescriptions was also observed in the medical wards. Meropenem was also the most used antibiotic in the ICUs (Annex 3).

\section{Ceftriaxone and Meropenem Extensive Use for Any Type of Indication or Infection}

The carbapenems were most frequently used to treat HAIs (Table 4), as well as the following antibiotics: meropenem, ceftriaxone, imipenem and cilastatin, and piperacillin (Annex 4). Fifty-six percent of the total prescriptions for HAIs were empirical, mainly for ceftriaxone, imipenem and cilastatin, and piperacillin (Annex 5). The empirical treatments for HAIs in the hospitals ranged from $52.3 \%$ to $85.0 \%$. The third-generation cephalosporins were mostly prescribed for patients with CAI in three of the hospitals (Table 4). Ceftriaxone, piperacillin, and sulfamethoxazole and trimethoprim were the most used antibiotics to treat CAIs. Ceftriaxone prescriptions accounted for almost half of total J01 prescriptions for CAIs in H4 (Annex 4). The overall proportion of empirical prescriptions for CAIs was 


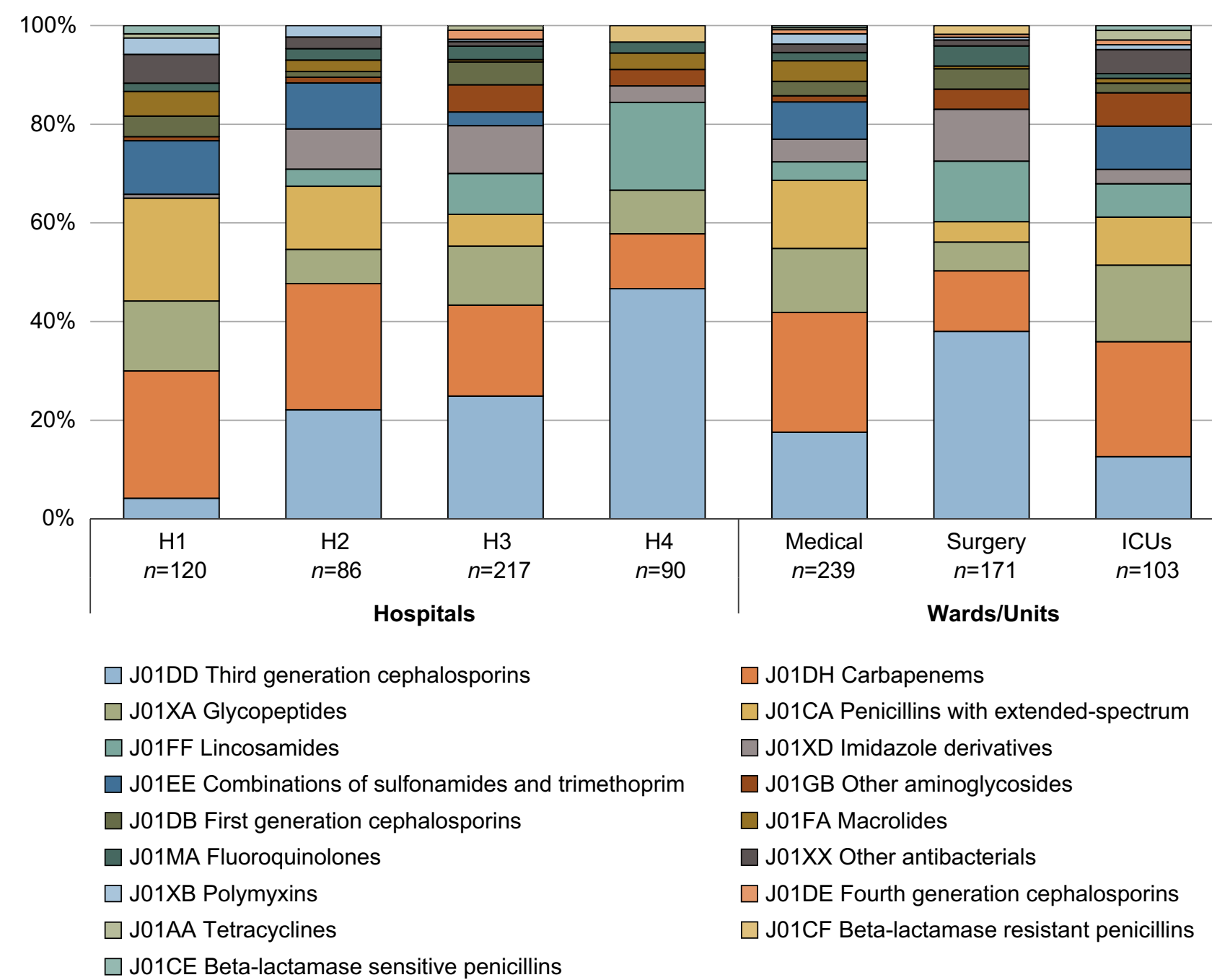

Figure I Percentage of J0I prescriptions at ATC level 4 in the hospitals and wards/units surveyed.

higher than for HAIs, mainly for ceftriaxone, piperacillin, and clarithromycin (Annex 6). Empirical prescriptions for CAI were also common in all hospitals, ranging from $66.7 \%$ to $88.6 \%$ (Table 4 ).

The third-generation cephalosporins, carbapenems, glycopeptides, and the combinations of sulfonamides and trimethoprim were the most used for medical prophylaxis (Table 4), as well as the following antibiotics: ceftriaxone, meropenem, vancomycin, and sulfamethoxazole and trimethoprim (Annex 4). The third-generation cephalosporins were also the most used for surgical prophylaxis (Table 4). Between $24.1 \%$ and $92.3 \%$ of total surgical prophylaxis prescriptions corresponded to ceftriaxone, which was predominantly prescribed in multiple doses for more than one day (Annex 4, 7).

The patients with pneumonia were treated mostly with ceftriaxone, piperacillin, meropenem, and vancomycin.
Whereas the antibiotics imipenem and cilastatin, meropenem, ertapenem, and clindamycin were commonly used for the patients with sepsis' treatment (Annex 8).

\section{Resistant Escherichia coli and ESKAPE Pathogens Were Frequently Identified in the Hospitals}

A wide variation in the proportion of patients with microbiological cultures was observed between the hospitals, ranging from $30.2 \%$ to $91.7 \%$. The number of species, genus or families of microorganisms isolated also varied considerably, from 11 to 81 per hospital. Gram-negative bacterial species/genus were frequently identified, which accounted for $48.8 \%$ to $91.3 \%$ of the total isolates. Positive isolates for E. coli, $P$. aeruginosa and K. pneumoniae were found in all hospitals (Table 5). 


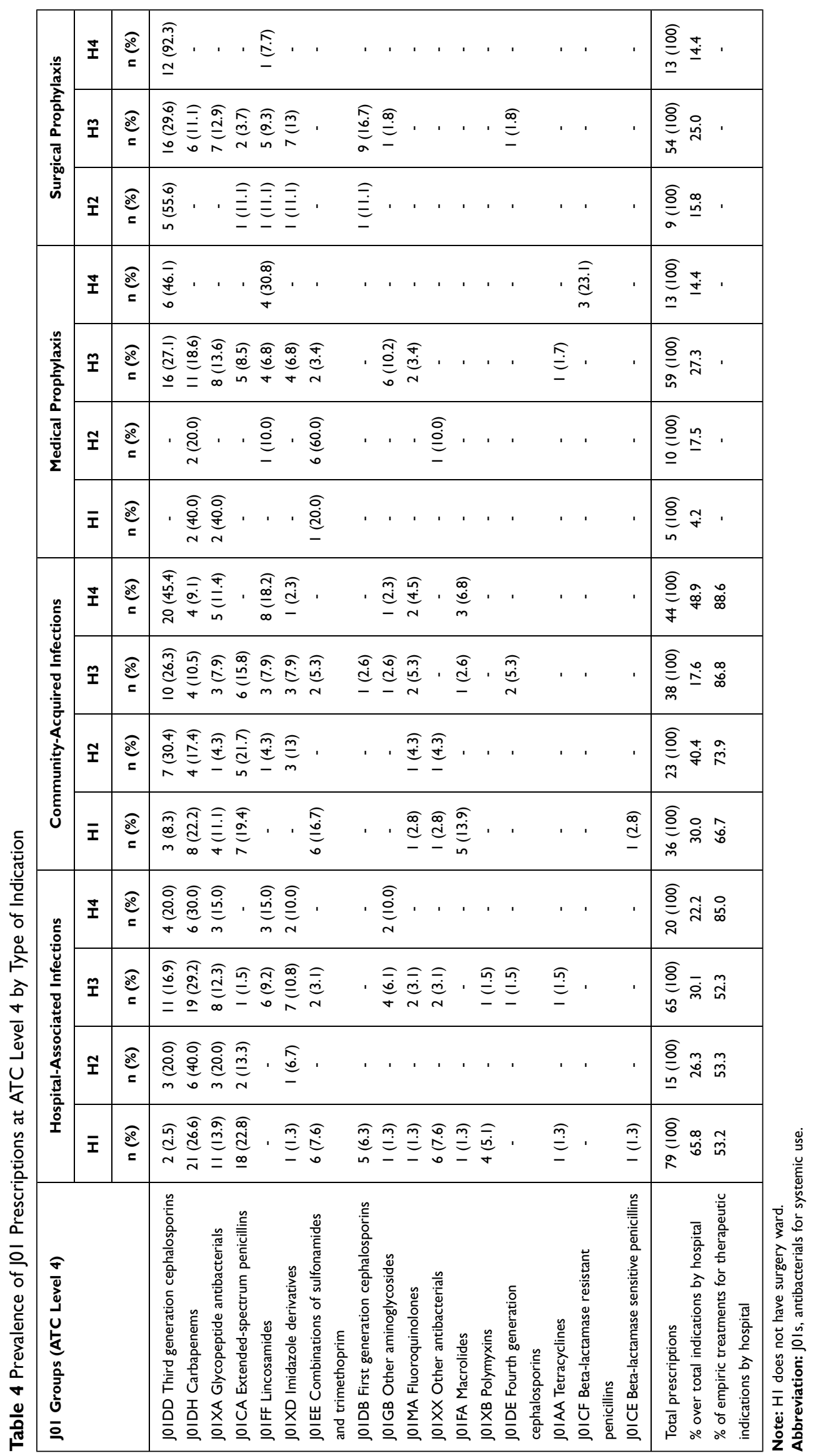


Table 5 General Characteristics of Isolations of Microorganisms in the Hospitals Surveyed

\begin{tabular}{|c|c|c|c|c|}
\hline & \multicolumn{4}{|c|}{ Hospitals } \\
\hline & $\mathbf{H I}$ & H2 & H3 & H4 \\
\hline & n (\%) & n (\%) & n (\%) & n (\%) \\
\hline Patients on AMs with Clinical Culture & 77 (91.7\%) & 42 (75.0\%) & 92 (78.0\%) & $19(30.2 \%)$ \\
\hline Number of Specimens for Culture & $n=199$ & $n=74$ & $n=159$ & $\mathbf{n}=\mathbf{2 9}$ \\
\hline $\begin{array}{l}\text { Microorganisms isolated } \\
\text { Gram negatives } \\
\text { - Escherichia coli } \\
\text { - Pseudomonas aeruginosa } \\
\text { - Klebsiella pneumoniae } \\
\text { - Other Citrobacter spp } \\
\text { - Klebsiella oxytoca } \\
\text { - Eikenella corrodens } \\
\text { - Gram-negative bacilli } \\
\text { - Enterobacter cloacae } \\
\text { - Stenotrophomonas maltophilia } \\
\text { - Other Gram-negative bacilli } \\
\text { - Prevotella spp } \\
\text { - Other Gram-negative cocci } \\
\text { - Other Enterobacter spp } \\
\text { - Other Klebsiella spp } \\
\text { - Salmonella spp, not specified } \\
\text { - Other Salmonella spp } \\
\text { - Acinetobacter baumannii } \\
\text { - Acinetobacter haemolyticus } \\
\text { - Burkholderia cepacia } \\
\text { - Other Pseudomonadaceae family } \\
\text { - Pseudomonadaceae family, not specified } \\
\text { - Morganella morganii }\end{array}$ & $\begin{array}{c}2 \mid(48.8) \\
7(\mid \mathrm{I} .3) \\
5(\mid \mathrm{I} .6) \\
2(4.6) \\
- \\
2(4.6) \\
- \\
- \\
- \\
2(4.6) \\
- \\
- \\
- \\
- \\
\text { I }(2.3) \\
\text { I }(2.3) \\
\text { I }(2.3) \\
- \\
- \\
- \\
- \\
- \\
-\end{array}$ & $\begin{array}{c}2 \mid(9 \mid .3) \\
8(34.8) \\
\text { I (4.3) } \\
3(\mid 3.0) \\
- \\
\text { I (4.3) } \\
3(\mid 3.0) \\
- \\
2(8.7) \\
- \\
2(8.7) \\
- \\
- \\
- \\
- \\
- \\
- \\
- \\
\text { I (4.3) } \\
- \\
- \\
- \\
-\end{array}$ & $\begin{array}{c}40(49.4) \\
\text { I4 (I7.5) } \\
3(3.7) \\
3(3.7) \\
5(6.2) \\
2(2.5) \\
\text { I }(1.2) \\
3(3.7) \\
- \\
- \\
- \\
2(2.5) \\
\text { I (I.2) } \\
\text { I (I.2) } \\
- \\
- \\
- \\
\text { I }(1.2) \\
- \\
\text { I (I.2) } \\
\text { I (I.2) } \\
\text { I (I.2) } \\
\text { I (I.2) }\end{array}$ & $\begin{array}{c}8(72.7) \\
4(36.4) \\
3(27.3) \\
1(9.1) \\
- \\
- \\
- \\
- \\
- \\
- \\
- \\
- \\
- \\
- \\
- \\
- \\
- \\
- \\
- \\
- \\
- \\
- \\
-\end{array}$ \\
\hline $\begin{array}{l}\text { Gram positives } \\
\text { - Enterococcus faecalis } \\
\text { - Staphylococcus epidermidis } \\
\text { - Staphylococcus aureus } \\
\text { - Enterococcus faecium } \\
\text { - Streptococcus spp } \\
\text { - Gram-positive cocci, not specified } \\
\text { - Clostridium difficile } \\
\text { - Other Clostridium } \\
\text { - Staphylococcus haemolyticus } \\
\text { - Enterococcus spp } \\
\text { - Actinomyces spp }\end{array}$ & $\begin{array}{l}\text { II }(25.6) \\
\text { I }(2.3) \\
\text { I }(2.3) \\
3(7.0) \\
5(11.6) \\
- \\
- \\
- \\
- \\
- \\
\text { I }(2.3) \\
-\end{array}$ & $\begin{array}{c}2(8.7) \\
- \\
- \\
- \\
- \\
- \\
- \\
2(8.7) \\
- \\
- \\
- \\
-\end{array}$ & $\begin{array}{c}36(44.4) \\
10(12.5) \\
8(10.0) \\
5(6.2) \\
3(3.7) \\
5(6.2) \\
2(2.5) \\
- \\
1(1.2) \\
1(1.2) \\
- \\
1(1.2)\end{array}$ & $\begin{array}{l}- \\
- \\
- \\
- \\
- \\
- \\
- \\
- \\
- \\
- \\
- \\
-\end{array}$ \\
\hline $\begin{array}{l}\text { Fungi } \\
\text { - Candida spp } \\
\text { - Aspergillus fumigatus } \\
\text { - Cryptococcus spp }\end{array}$ & $\begin{array}{l}\text { II }(25.6) \\
9(20.9) \\
2(4.6) \\
-\end{array}$ & $\begin{array}{l}- \\
- \\
- \\
-\end{array}$ & $\begin{array}{c}4(5.0) \\
4(5.0) \\
- \\
-\end{array}$ & $\begin{array}{c}3(27.3) \\
2(18.2) \\
- \\
1(9.1)\end{array}$ \\
\hline Total isolations & $43(100)$ & $23(100)$ & $81(100)$ & II (100) \\
\hline
\end{tabular}


Table 6 Species/Genus of Bacteria with Antimicrobial Susceptibility Testing (AST) in the Hospitals Surveyed

\begin{tabular}{|c|c|c|}
\hline Hospitals & $\begin{array}{l}\text { Species/Genus of Bacteria with } \\
\text { Resistance Results }\end{array}$ & J0 Is at ATC Level 5 (>I AST Result) \\
\hline $\mathrm{HI}$ & $\begin{array}{l}\text { Escherichia coli } \\
\text { Pseudomonas aeruginosa } \\
\text { Enterococcus faecium } \\
\text { Staphylococcus epidermidis } \\
\text { Klebsiella pneumoniae } \\
\text { Klebsiella oxytoca } \\
\text { Other Klebsiella spp } \\
\text { Salmonella spp, not specified } \\
\text { Other Salmonella spp }\end{array}$ & 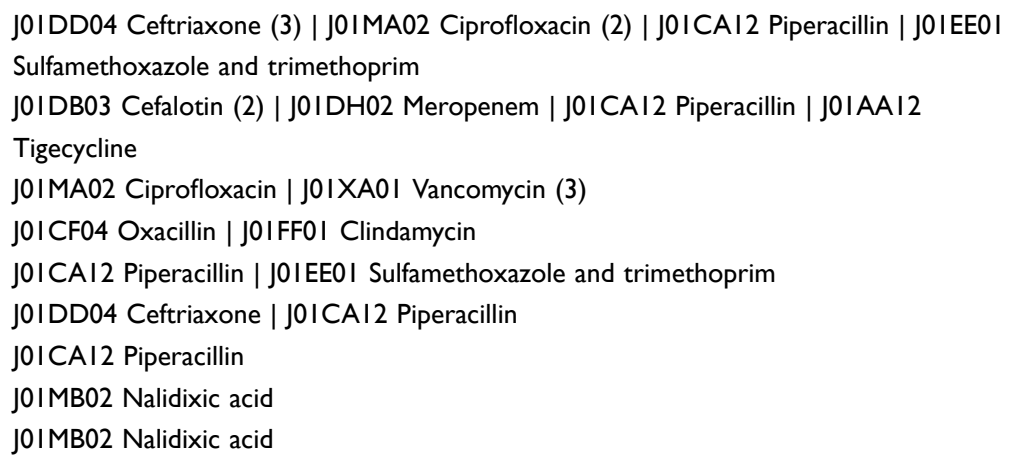 \\
\hline $\mathrm{H} 3$ & $\begin{array}{l}\text { Staphylococcus epidermidis } \\
\text { Other Citrobacter spp } \\
\text { Escherichia coli } \\
\text { Staphylococcus haemolyticus } \\
\text { Staphylococcus aureus } \\
\text { Enterococcus faecalis } \\
\text { Klebsiella oxytoca } \\
\text { Streptococcus spp } \\
\text { Klebsiella pneumoniae } \\
\text { Morganella morganii }\end{array}$ & $\begin{array}{l}\text { J0IFA0I Erythromycin | J0 IGB03 Gentamicin (3) | J0 ICF04 Oxacillin | J0 IFF0 I } \\
\text { Clindamycin (6) } \\
\text { J0IDD02 Ceftazidime (2) | J0IDD04 Ceftriaxone (2) | J0IMA02 Ciprofloxacin } \\
\text { J0IDD04 Ceftriaxone (5) } \\
\text { J0IMA02 Ciprofloxacin | J0IGB03 Gentamicin | J0IMAI2 Levofloxacin } \\
\text { J0IMA02 Ciprofloxacin | J0 IFF0 I Clindamycin } \\
\text { J0IGB03 Gentamicin (2) } \\
\text { J0IDD04 Ceftriaxone (2) } \\
\text { J0IFF0I Clindamycin } \\
\text { J0IDD04 Ceftriaxone } \\
\text { J0IMA02 Ciprofloxacin }\end{array}$ \\
\hline $\mathrm{H} 4$ & $\begin{array}{l}\text { Escherichia coli } \\
\text { Klebsiella pneumoniae } \\
\text { Pseudomonas aeruginosa }\end{array}$ & $\begin{array}{l}\text { J0ICA0I Ampicillin (4) | JOIDE0 I Cefepime (3) | J0IDD04 Ceftriaxone | J0IMA02 } \\
\text { Ciprofloxacin | J0ICR04 Sultamicillin (3) } \\
\text { J0ICA0I Ampicillin | JOIDE0I Cefepime | J0 ICR04 Sultamicillin } \\
\text { J0ICAI2 Piperacillin | JOIDF0 I Aztreonam | Pan-drug resistance }\end{array}$ \\
\hline
\end{tabular}

Notes: Data on the specific AST methods performed were not available in clinical records. $\mathrm{H} 2$ had no data of AST.

Abbreviations: AST, antimicrobial susceptibility testing; $\mathrm{JOIs}$, antibacterials for systemic use.

Data on AST were only found in three hospitals $(\mathrm{H} 1, \mathrm{H} 3$, H4). Multidrug-resistant E. coli, and at least two species of ESKAPE pathogens: $K$. pneumoniae, $P$. aeruginosa, $S$. aureus or E. faecium were identified in all hospitals with AST data (Table 6). Aztreonam-resistant and pandrogo-resistant $P$. aeruginosa strains were also identified (H4), as well as vancomycin-resistant E. faecium strains (H1). E. coli and ESKAPE pathogens were mostly resistant to ceftriaxone, ampicillin, piperacillin, and ciprofloxacin.

\section{Discussion}

The PPSs showed variability in the prevalence of AMs use among the hospitals surveyed (47.1\% to $91.3 \%)$. This variability could possibly be related to differences in the case-mix, and particular structural characteristics of the hospitals, such as the type and balance of medical specialties, that are not part of the data included in the PPS protocol. No matter that variability, our data revealed a wide use of AMs in the hospitals surveyed exceeding those used in hospitals of Europe (29.0\%), Latin America (36.8\%), and US (49.9\%). ${ }^{22,23,29}$ Furthermore, our results were higher than those reported previously by other tertiary care Mexican hospitals $(59.3 \%, 51.5 \%) .{ }^{14,30}$ Our findings also describe the extensive use of broadspectrum J01s such as ceftriaxone, meropenem, vancomycin, piperacillin, and clindamycin. These results are consistent with trends in the use of these antibiotics in Mexico and Latin America. ${ }^{13,14,17,23,30,31}$ Nevertheless, we identified even greater use proportions of these five antibiotics than those commonly found in Latin America, mainly ceftriaxone and meropenem. ${ }^{23}$ These last two antibiotics belong to the WHO "Watch" group, only recommended for a specific and limited number of indications due to their elevated risk of resistant-bacteria selection. ${ }^{32}$ Thus, the fact that almost half of the antibiotics used in 
one of the hospitals surveyed (H4) were ceftriaxone, suggests that at least a proportion of these prescriptions were inappropriate. Furthermore, ceftriaxone was predominantly prescribed in multiple doses for more than one day for surgical prophylaxis, contrary to the conventional recommendation of a single dose of cephalosporins (eg, cefazolin), $1 \mathrm{~h}$ before surgical incision for most surgical procedures. ${ }^{33}$ Besides, although the microbiology laboratories' role and rate of antimicrobial cultures per patient varied widely among the hospitals surveyed, none of them appears to have used the culture results and AST data for de-escalation of the antibiotic therapies, as recommended to reduce detrimental effects on patients as well as to minimize the development of AMR. ${ }^{34}$

All the above could represent a common scenario associated to the recurrent identification of MDR E. coli and ESKAPE pathogens in the hospitals surveyed, as well as in other Mexican hospitals. ${ }^{16,17,35,36}$

We consider that our findings could be partly explained by the absence of formally established ASPs in the hospitals surveyed. ASPs consist of a variety of systematic activities and interventions aimed to optimize the antimicrobial use. The core elements of this type of program have been defined, ${ }^{37}$ and a set of possible activities identified and positively analyzed in systematic reviews. ${ }^{38,39}$ Professionals associations and both national and international health agencies strongly support the implementation of these programs. ${ }^{37,40,41}$ In recent years, the WHO has pushed forward this approach for low- and middle-income countries by publishing guidelines for implementing ASPs in these settings. ${ }^{41}$ However, the hospitals surveyed did not have guidelines for antimicrobial prescribing or formal evidence of other possible interventions to improve the antimicrobial use. Therefore, we consider it urgent to plan and implement ASP programs in these Mexican hospitals, or at least devise and ensure the formal implementation of some of the interventions that have proven effective for controlling and improving the antimicrobial use. These interventions could include implementing international or local antimicrobial prescription guidelines, educational, audit, and feedback strategies, managing the use of broad-spectrum antibiotics, improving the quality of surgical prophylaxis, and reinforcing the implementation of planned duration treatments, among others. ${ }^{12,38,39,42-45}$ The absence of a defined strategy and plan for improving the antimicrobial use at the health system level is not helping either. We may argue that the absence of ASPs in Mexican public hospitals, like the ones we have studied, could be partially due to the lack of an official, explicit, and operative policy to improve the antimicrobial use in the Mexican health-care system.

The results of this study show the potential usefulness of raising of awareness about routine AM prescription at the patient level. The strength of our results is based on the data collection uniformity, achieved by conducting pilot studies in the four hospitals prior to the PPSs. ${ }^{25}$ However, our study is not exempt of limitations. Some of them are intrinsic to the PPS method: data are transversal but collected at different months of the year (between August and December); long-term treatments may be overrepresented; and it is not possible to identify the patients' cases combination, changes in the incidence and prevalence of different infections, or institutional factors, all of which may influence the antimicrobial prescribing patterns. Our findings also do not reflect the prescribing practices of other hospitals in Mexico, particularly in pediatric' hospitals.

\section{Conclusions}

Our PPSs reveal the extensive use of broad-spectrum antibiotics for systemic use for empirical treatments, with no planned duration or review dates, and the excessive and prolonged use of certain antibiotics (ie, ceftriaxone). Furthermore, we identified the recurrent presence of E. coli and ESKAPE pathogens, which many of them are resistant to the antibiotics frequently used in the hospitals surveyed, suggesting that a vicious cycle of prescribers used to particular patterns of antibiotic prescription has been created.

The first step to improve the antimicrobial prescribing behavior in hospitals is to provide enough evidence of their prescription patterns aiming at the identification of objectives for optimization on antibiotic use. Thereby, can be prioritized specific aims to optimize the day-to-day antibiotic prescribing on patients. PPSs at the hospital or at priority wards/units' level could be a useful method for this purpose.

\section{Abbreviations}

PPSs, point prevalence surveys; AMs, antimicrobials; J01s, antibiotics for systemic use; AMR, antimicrobial resistance; ESKAPE, Enterococcus faecium, Staphylococcus aureus, Klebsiella pneumoniae, Acinetobacter baumannii, Pseudomonas aeruginosa, and Enterobacter spp; WHO, World Health Organization; ASPs, antimicrobial stewardship programs; WPPS, WHO methodology for point prevalence survey on antibiotic use in hospitals; H1, hospital 1; H2, 
hospital 2; H3, hospital 3; H4, hospital 4; ICUs, intensive-care units; HAIs, hospital-associated infections; CAIs, community-acquired infections; AST, antimicrobial susceptibility testing; ATC, Anatomical Therapeutic Chemical classification system; MDR, multidrug-resistant.

\section{Ethical Approval}

The protocol of this study was approved by the research ethics committee of the National Institute of Public Health of Mexico (RF. 17CEI00420160708). This study did not include any interaction with patients. Informed consents to review the patient's medical records were not required since all collected patients' data was anonymous. All the authors assume the obligation to protect the individual' privacy and maintain the confidentiality of the patients' data in compliance with the Declaration of Helsinki, and the Regulation of the General Health Law on Health Research of Mexico.

\section{Acknowledgments}

The authors thank the Mexican National Council on Science and Technology (CONACYT) for supporting this work [grant number: 290618]. We also thank Kathleen Lanette Chavarría Guzmán, Diana María Ramírez Fernández and Antonia Indahita Rodríguez Martínez for conducting the data collection of this study.

\section{Disclosure}

The authors report no conflicts of interest in this work.

\section{References}

1. Holmes AH, Moore LSP, Sundsfjord A, et al. Understanding the mechanisms and drivers of antimicrobial resistance. Lancet. 2016;387(10014):176-187. doi:10.1016/S0140-6736(15)00473-0

2. Founou RC, Founou LL, Essack SY. Clinical and economic impact of antibiotic resistance in developing countries: a systematic review and meta-analysis. PLoS One. 2017;12(12):1-18. doi:10.1371/journal. pone. 0189621

3. Gulen TA, Guner R, Celikbilek N, Keske S, Tasyaran M. Clinical importance and cost of bacteremia caused by nosocomial multi drug resistant Acinetobacter baumannii. Int J Infect Dis. 2015;38:32-35. doi:10.1016/j.ijid.2015.06.014

4. Centres for Disease Control and Prevention UD of $\mathrm{H}$ and HS. Antibiotic Resistance: A Global Threat. World Health Organization; 2013.

5. O'Neil J. Review on antibiotic resistance. Antimicrobial resistance: tackling a crisis for the health and wealth of nations. Health Wealth Nations. 2014;(December):1-16.

6. World Health Organization. Global action plan on antimicrobial resistance; 2015. Available from: https://www.who.int/iris/bitstream/ 10665/193736/1/9789241509763_eng.pdf?ua=1. Accessed October 20, 2021.
7. Fridkin S, Baggs J, Fagan R, et al. Vital signs: improving antibiotic use among hospitalized patients. MMWR Morb Mortal Wkly Rep. 2014;63(9):194-200.

8. Tamma PD, Avdic E, Li DX, Dzintars K, Cosgrove SE. Association of adverse events with antibiotic use in hospitalized patients. JAMA Intern Med. 2017;177(9):1308-1315. doi:10.1001/jamainternmed.20 17.1938

9. Marchaim D, Chopra T, Bhargava A, et al. Recent exposure to antimicrobials and carbapenem-resistant Enterobacteriaceae: the role of antimicrobial stewardship. Infect Control Hosp Epidemiol. 2012;33(8):817-830. doi:10.1086/666642

10. Paterson DL. "Collateral damage" from cephalosporin or quinolone antibiotic therapy. Clin Infect Dis. 2004;38(Supp14):341-345. doi: $10.1086 / 382690$

11. Rice LB. Federal funding for the study of antimicrobial resistance in nosocomial pathogens: no ESKAPE. J Infect Dis. 2008;197 (8):1079-1081. doi:10.1086/533452

12. Davey P, Marwick CA, Scott CL, et al. Interventions to improve antibiotic prescribing practices for hospital inpatients. Cochrane Database Syst Rev. 2017;(2):Art. No.: CD003543. doi:10.1002/ 14651858.CD003543.pub4

13. Wirtz VJ, Dreser A, Gonzales R. Trends in antibiotic utilization in eight Latin American countries, 1997-2007. Rev Panam Salud Publica/Pan Am J Public Health. 2010;27(3):219-225. doi:10.1590/ S1020-49892010000300009

14. Huerta-Gutiérrez R, Braga L, Camacho-Ortiz A, et al. One-day point prevalence of healthcare-associated infections and antimicrobial use in four countries in Latin America. Int J Infect Dis. 2019;86:157-166. doi:10.1016/j.ijid.2019.06.016

15. Aguilar-Rodea B, Cureño-Diaz M, Alvarez-Montero F, ValdesCastro R, Valdez-Vázquez R, Figueroa-Moreno R. Epidemiology of healthcare-associated infections at a General Hospital in Mexico City: 2013-2014. Open Forum Infect Dis. 2015;2(suppl_1). doi:10. 1093/ofid/ofv133.1269

16. Garza-González E, Morfín-Otero R, Mendoza-Olazarán S, et al. A snapshot of antimicrobial resistance in Mexico. Results from 47 centers from 20 states during a six-month period. PLoS One. 2019;14 (3):1-13. doi:10.1371/journal.pone.0209865

17. Miranda-Novales MG, Flores-Moreno K, López-Vidal Y, et al. Antimicrobial resistance and antibiotic consumption in Mexican hospitals. Salud Publica Mex. 2020;62(1):42-49.

18. Álvarez de Iturbe I, Chirino-Barceló Y, López López A, Zamorano Jiménez CA, Nava Frías M. Uso de la profilaxis antimicrobiana en el Departamento de Cirugía en un hospital de tercer nivel de atención. An Med. 2013;58(4):247-252.

19. Solís-Téllez H, Mondragón-Pinzón EE, Ramírez-Marino M, et al. Epidemiologic analysis: prophylaxis and multidrug-resistance in surgery. Rev Gastroenterol México. 2017;82(2):115-122. doi:10.10 16/j.rgmxen.2016.12.002

20. Palacios-Saucedo G Del C, de la Garza-camargo M, Briones-Lara E, et al. Evaluación del uso de antibióticos e impacto de una intervención dirigida a modificar la conducta prescriptiva en profilaxis quirúrgica en 6 hospitales del área metropolitana de Monterrey. Cir Cir. 2017;85(6):459-470. doi:10.1016/j.circir.2016.10.033

21. Ansari F, Erntell M, Goossens H, Davey P. The European surveillance of antimicrobial consumption (ESAC) point-prevalence survey of antibacterial use in 20 European hospitals in 2006. Clin Infect Dis. 2009;49(10):1496-1504. doi:10.1086/644617

22. Zarb P, Amadeo B, Muller A, et al. Identification of targets for quality improvement in antimicrobial prescribing: the web-based ESAC point prevalence survey 2009. J Antimicrob Chemother. 2011;66(2):443-449. doi:10.1093/jac/dkq430

23. Versporten A, Zarb P, Caniaux I, et al. Antimicrobial consumption and resistance in adult hospital inpatients in 53 countries: results of an internet-based global point prevalence survey. Lancet Glob Health. 2018;6(6):e619-e629. doi:10.1016/S2214-109X(18)30186-4 
24. World Health Organization. WHO methodology for point prevalence survey on antibiotic use in hospitals, version 1.1; 2018. Available from: https://www.who.int/medicines/access/antimicrobial_resis tance/WHO-EMP-IAU-2018_01/en/. Accessed October 20, 2021.

25. Zumaya-Estrada FA, Alpuche-Aranda CM, Saturno-Hernandez PJ. The WHO methodology for point prevalence surveys on antibiotics use in hospitals should be improved: lessons from pilot studies in four Mexican hospitals. Int $J$ Infect Dis. 2021;108:13-17. doi:10.1016/j.ijid.2021.04.079

26. World Health Organization. International language for drug utilization research; 2020. Available from: https://www.whocc.no/. Accessed October 20, 2021.

27. IBM. IBM SPSS statistics for windows; 2017.

28. GraphPad. GraphPad QuickCalcs; 2021. Available from: https:// www.graphpad.com/quickcalcs/confInterval1//. Accessed October $20,2021$.

29. Magill SS, Edwards JR, Beldavs ZG, et al. Prevalence of antimicrobial use in us acute care hospitals, May-September 2011. JAMA. 2014;312(14):1438-1446. doi:10.1001/jama.2014.12923

30. Soria-Orozco M, Padrón-Salas A, González-mercado J de J, et al. Prevalencia de uso de antimicrobianos entre pacientes hospitalizados en áreas no críticas en un hospital universitario de México. Salud Publica Mex. 2017;59(5):504-505. doi:10.21149/8465

31. Porto APM, Goossens H, Versporten A, Costa SF. Global point prevalence survey of antimicrobial consumption in Brazilian hospitals. J Hosp Infect. 2020;104(2):165-171. doi:10.1016/j.jhin.20 19.10 .016

32. World Health Organization. The 2019 WHO AWaRe classification of antibiotics for evaluation and monitoring of use; 2019. Available from: https://www.who.int/publications/i/item/WHOEMPIAU2019. 11. Accessed October 20, 2021.

33. Bratzler DW, Dellinger EP, Olsen KM, et al. Clinical practice guidelines for antimicrobial prophylaxis in surgery. Am J Health Pharm. 2013;70(3):195-283. doi:10.2146/ajhp120568

34. Lakbar I, De Waele JJ, Tabah A, Einav S, Martin-Loeches I, Leone M. Antimicrobial de-escalation in the ICU: from recommendations to level of evidence. Adv Ther. 2020;37(7):3083-3096. doi:10.1007/s12325-020-01390-2

35. Rawat D, Nair D. Extended-spectrum $\beta$-lactamases in gram negative bacteria. J Glob Infect Dis. 2010;2(3):263-274. doi:10.4103/0974$777 \mathrm{X} .68531$
36. Garza-González E, Bocanegra-Ibarias P, Bobadilla-Del-Valle M, et al. Drug resistance phenotypes and genotypes in Mexico in representative gram-negative species: results from the infivar network. PLoS One. 2021;16(3):e0248614. doi:10.1371/journal.pone.0248614

37. CDC. The core elements of hospital antibiotic stewardship programs: 2019; 2019. Available from: https:/www.cdc.gov/antibiotic-use/coreelements/hospital.html. Accessed October 20, 2021.

38. Wagner B, Filice GA, Drekonja D, et al. Antimicrobial stewardship programs in inpatient hospital settings: a systematic review. Infect Control Hosp Epidemiol. 2014;35(10):1209-1228. doi:10.1086/ 678057

39. Karanika S, Paudel S, Grigoras C, Kalbasi A, Mylonakis E. Systematic review and meta-analysis of clinical and economic outcomes from the implementation of hospital-based antimicrobial. Antimicrob Agents Chemother. 2016;60(8):4840-4852. doi:10.1128/ AAC.00825-16

40. Barlam TF, Cosgrove SE, Abbo LM, et al. Implementing an antibiotic stewardship program: guidelines by the Infectious Diseases Society of America and the Society for Healthcare Epidemiology of America. Clin Infect Dis. 2016;62:51-77. doi:10.1093/cid/ciw118

41. World Health Organization. Antimicrobial stewardship programmes in health-care facilities in low- and middle-income countries. A practical toolkit. 2019.

42. Wilkinson A, Ebata A, Macgregor H. Interventions to reduce antibiotic prescribing in LMICs: a scoping review of evidence from human and animal health systems. Antibiotics. 2019;8(1). doi:10. 3390/antibiotics 8010002

43. Oliveira I, Rego C, Semedo G, et al. Systematic review on the impact of guidelines adherence on antibiotic prescription in respiratory infections. Antibiotics. 2020;9(9):1-19. doi:10.3390/antibiotics9090 546

44. Segala FV, Murri R, Taddei E, et al. Antibiotic appropriateness and adherence to local guidelines in perioperative prophylaxis: results from an antimicrobial stewardship intervention. Antimicrob Resist Infect Control. 2020;9(1):1-6. doi:10.1186/s13756-020-00814-6

45. Vercheval C, Gillet M, Maes N, et al. Quality of documentation on antibiotic therapy in medical records: evaluation of combined interventions in a teaching hospital by repeated point prevalence survey. Eur J Clin Microbiol Infect Dis. 2016;35(9):1495-1500. doi:10.1007/ s10096-016-2690-0
Infection and Drug Resistance

\section{Publish your work in this journal}

Infection and Drug Resistance is an international, peer-reviewed openaccess journal that focuses on the optimal treatment of infection (bacterial, fungal and viral) and the development and institution of preventive strategies to minimize the development and spread of resistance. The journal is specifically concerned with the epidemiology of antibiotic resistance and the mechanisms of resistance development and diffusion in both hospitals and the community. The manuscript management system is completely online and includes a very quick and fair peerreview system, which is all easy to use. Visit http://www.dovepress.com/ testimonials.php to read real quotes from published authors. 